\title{
The environs of the $\mathrm{H}$ II region Gum 31
}

\author{
C. Cappa ${ }^{1,2, \star}$, V. S. Niemela ${ }^{1,3, \star \star}$, R. Amorín ${ }^{4}$, and J. Vasquez ${ }^{1,2 . \star \star \star}$
}

\author{
1 Facultad de Ciencias Astronómicas y Geofísicas. Universidad Nacional de La Plata. Paseo del Bosque s/ı. 1900 La Plata. Argentina \\ e-mail: ccappa@fcaglp.fcaglp.unlp.edu.ar \\ 2 Instituto Argentino de Radioastronomía, C.C. 5, 1894 Villa Elisa, Argentina \\ 3 Instituto de Astrofísica de La Plata, La Plata, Argentina \\ ${ }^{4}$ Instituto de Astrofísica de Canarias, Spain
}

Received 26 December 2006 / Accepted 3 October 2007

\section{ABSTRACT}

\begin{abstract}
Aims. We analyze the distribution of the interstellar matter in the environs of the Hn region Gum 31, excited by the open cluster NGC 3324, located in the complex Carina region, with the aim of investigating the action of the massive stars on the surrounding nentral material.

Methods. We use neutral hydrogen $21-\mathrm{cm}$ line data, radio continuum images at $0.843,2.4$ and $4.9 \mathrm{GHz}$. ${ }^{12} \mathrm{CO}(1-0)$ observations. and IRAS and MSX infrared data.

Results. Adopting a distance of $3 \mathrm{kpc}$ for the $\mathrm{Hn}$ region and the ionizing cluster, we derived an electron density of $33 \pm 3 \mathrm{~cm}^{-3}$ and an ionized mass of $(3.3 \pm 1.1) \times 10^{3} M_{\odot}$ based on the radio continuum data at $4.9 \mathrm{GHz}$. The $\mathrm{H} 121-\mathrm{cm}$ line images revealed an $\mathrm{H} 1$ shell surrounding the $\mathrm{Hn}$ region. The $\mathrm{Hl}$ structure is $10.0 \pm 1.7 \mathrm{pc}$ in radius, has a neutral mass of $1500 \pm 500 M_{\Omega}$, and is expanding at $11 \mathrm{~km} \mathrm{~s}^{-1}$. The associated molecular gas amounts to $(1.1 \pm 0.5) \times 10^{5} M_{\circ}$. being its volume density of about $350 \mathrm{~cm}^{-3}$. This molecular shell could represent the remains of the clond where the young open cluster NGC 3324 was born or conld have originated by the shock front associated with the Hn region. The difference between the ambient density and the electron density of the Hn region suggests that the Hn region is expanding.

The distributions of the ionized and molecular material, along with that of the emission in the MSX band A. suggest that a photodissociation region has developed at the interface between the ionized and molecular gas. The copions UV photon flux from the early type stars in NGC 3324 keeps the Hn region ionized.

The characteristics of a relatively large number of the IRAS, MSX, and 2MASS point sources projected onto the molecular envelope are compatible with protostellar candidates, showing the presence of active star forming regions. Very probably, the expansion of the $\mathrm{Hn}$ region has triggered stellar formation in the molecular shell.
\end{abstract}

Key words. ISM: Hn regions - ISM: individual objects: Gum 31 - stars: early-type - stars: individual: HD 92206 - ISM : bubbles

\section{Introduction}

The interstellar medium associated with the birth place of massive stars, such as $\mathrm{O}$ or early $\mathrm{B}$-type stars, is made up of dense giant molecular clouds. Massive stars are characterized by intense photon fluxes which ionize and photodissociate the surrounding material. An H II region is a direct consequence of the high rate of Lyman continuum luminosity. Initially, the H II region is a small, high deusity region, commonly named ultra-compact H II region (UC HII, Wood \& Churchwell 1989). If the photon flux rate of the massive star is sufficiently high, the $\mathrm{H}$ II region evolves into a normal $\mathrm{H}$ II region.

A neutral shell encircles the HII region during the expanding phase (e.g. Spitzer 1978). The presence of these neutral shells is observed in the H I $21 \mathrm{-cm}$ line emission distribution (e.g. Deharveng et al. 2003). Molecular line studies have allowed the identification of molecular gas following the outer borders of $\mathrm{H}$ II regions, indicating the presence of photodissociation regions (PDRs). Deharveng et al. (2005) have detected these PDRs in a number of H II regious.

\footnotetext{
* Member of Carrera del Investigador, CONICET, Argentina.

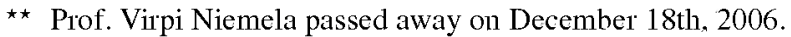

$\star \star \star$ Fellow of CONICET, Argentina.
}

In the present study, we analyze the distribution of the ionized and neutral material associated with the $\mathbf{H}$ II region Gum 31 (Gum 1955) based on H I 21-cm line emission data, radio continuum information at different frequencies, and IR and molecular data.

The HII region Gum 31 is about $15^{\prime}$ in size and approximately circular in shape (Fig. 1). It is located at $(l, b)=\left(286^{\circ} 12^{\prime}\right.$, $\left.-0^{\circ} 12^{\prime}\right)$ in the complex region of Carina and is considered a member of the Car OB 1 association. The SuperCOSMOS image (Parker et al. 2005) shows a quite inhomogeneous H II region, with a sharp and bright rim towards lower galactic longitudes and lower galactic latitudes, looking fainter and more diffuse towards higher galactic longitudes.

Based on data of the radio recombination lines (RRLs) $\mathrm{H} 109 \alpha$ and $\mathrm{H} 110 \alpha$ at $5 \mathrm{GHz}$, Caswell \& Haynes (1987) found that the LSR velocity of the ionized gas in Gum 31 is $-18 \mathrm{~km} \mathrm{~s}^{-1}$, similar to the velocities of other H II regions in the area of the Car OB1 association (Georgelin et al. 1986), and derived an electrou temperature $T_{\mathrm{e}}=7100 \mathrm{~K}$. They estimated a flux density $S_{5 \mathrm{GHz}}=35 \mathrm{Jy}$.

The excitation sources of the HII region Gum 31 are the OB star members of the open cluster NGC 3324. The brightest star in this cluster is HD 92206, which is the visual double star IDS 10336-5806 in the Index catalogue (Jeffers et al. 1963) with a 1 mag fainter companion (HD 92206B) placed 5 " to the 


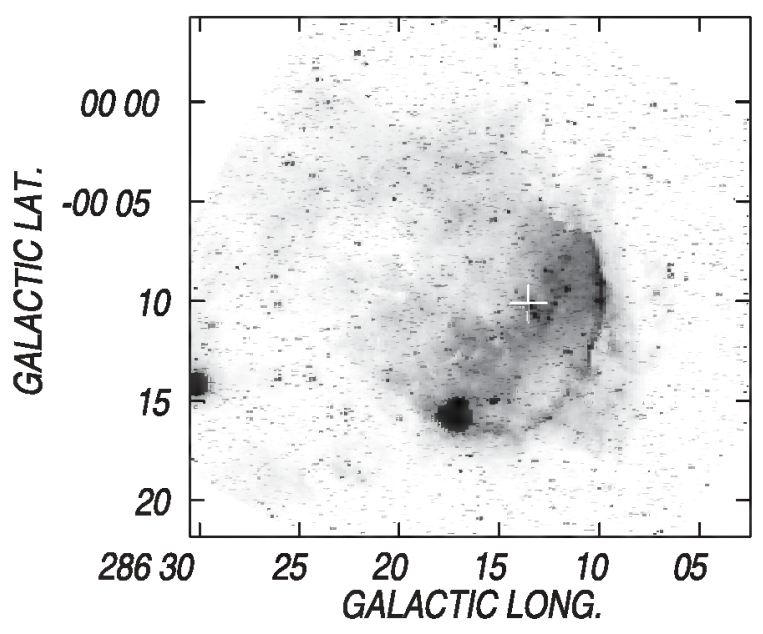

Fig. 1. SuperCOSMOS image showing the HII region Gum 31. The cross marks the position of the multiple system HD 92206. The intensity units are arbitrary.

East. Another bright cluster member is located $35^{\prime \prime}$ to the SW. This star is $\mathrm{CD}-57^{\circ} 3378$, also referred to as $\mathrm{HD} 92206 \mathrm{C}$ in the literature.

The three brightest stars in NGC 3324 have published spectral types. Both HD 92206A and B are classified as O6.5V, and HD $92206 \mathrm{C}$ as O9.5V by Mathys (1988). Walborn (1982) classifies HD 92206 A as O6.5V(n) and the component C as O8.5Vp.

Moffat \& Vogt (1975) first carried out photometric observations of about 12 stars in the cluster and estimated a color excess $E(B-V) \sim 0.45 \pm 0.05 \mathrm{mag}$ and a distance $d=3.3 \mathrm{kpc}$. Clariá (1977), using $U B V$ photometry, confirmed previous results by Moffat \& Vogt and estimated $d=3.1 \mathrm{kpc}$. Vazquez $\&$ Feinstein (1990) derived a distance $d=3.6 \mathrm{kpc}$ for the cluster from $U B V R I$ photometry. More recently, Carraro et al. (2001) found about 25 new possible cluster members and derived $d=3.0 \pm 0.1 \mathrm{kpc}$. On the other hand, distance estimates for Car OB 1 are in the range $1.8-2.8 \mathrm{kpc}$ (Walborn 1995). Bearing in mind these results we adopted $d=3.0 \pm 0.5 \mathrm{kpc}$ for both Gum 31 and the ionizing cluster.

Carraro et al. (2001) find evidence for pre-main sequence members beginning at about late B spectral type, which suggests an extremely young age for NGC $3324\left(\leq(2-3) \times 10^{6} \mathrm{yr}\right)$. The Otype members would be stars recently arrived on the Zero Age Main Sequence.

\section{Data sets}

\subsection{Radio data sets}

We analyzed the radio continuum emission in the region of Gum 31 using data obtained at $0.843,2.42$ and $4.85 \mathrm{GHz}$, which were extracted from the Sydney University Molonglo Sky Survey (SUMSS) (see Sadler \& Hunstead 2001, for a description), the survey by Duncan et al. (1995), and the Parkes-MITNRAO (PMN) Southern Radio Survey (see Condon et al. 1993, for a complete description of this survey), respectively.

We used H I data from the Southern Galactic Plane Survey (SGPS) to analyze the neutral gas distribution in the environs of Gum 31. These data were obtained with the Australia Telescope Compact Array (ATCA) and the Parkes Radiotelescope (short spacing information). A Hanning smoothing (Rohlfs 1986) was applied to the HI data to improve the signal to noise ratio.
Table 1. Radio data: relevant parameters.

\begin{tabular}{lc}
\hline \hline Radio continuum at $4.85 \mathrm{GHz}$ & \\
\hline Angular resolution & 5.0 \\
rms noise & $10 \mathrm{mJy} \mathrm{beam}^{-1}$ \\
\hline Radio continutum at $2.4 \mathrm{GHz}$ & \\
\hline Angular resolution & $10^{\prime} .4$ \\
Ims noise & $12 \mathrm{mJy} \mathrm{beam}^{-1}$ \\
\hline Radio continuum at $0.843 \mathrm{GHz}$ & \\
\hline Angular resolution & $43^{\prime \prime} \times 51^{\prime \prime}$ \\
rms noise & $1 \mathrm{mJy} \mathrm{beam}^{-1}$ \\
\hline H I data & \\
\hline Synthesized beam & $2 ! 4 \times 2^{\prime} \cdot 1$ \\
Number of channels & 256 \\
Velocity coverage & $(-190,+230) \mathrm{km} \mathrm{s}^{-1}$ \\
Velocity resolution & $1.64 \mathrm{~km} \mathrm{~s}^{-1}$ \\
RMS noise level & $1.6 \mathrm{~K}^{\text {I2CO(1-0) NANTEN data }}$ \\
\hline Angular resolution & $2 ! 7$ \\
Velocity coverage & $(-50 .+50) \mathrm{km} \mathrm{s}^{-1}$ \\
Velocity resolution & $0.2 \mathrm{~km} \mathrm{~s}^{-1}$ \\
RMS noise level & $1.0 \mathrm{~K}$ \\
\hline
\end{tabular}

A description of this survey can be found in McClure-Griffiths et al. (2005).

The distribution of the molecular material in the region was studied using ${ }^{12} \mathrm{CO}(1-0)$ line data at $115 \mathrm{GHz}$ obtained with the NANTEN $4 \mathrm{~m}$ telescope of Nagoya University at Las Campanas Observatory of the Carnegie Institution of Washington, and published by Yonekura et al. (2005). Observational parameters for these data were taken from Yonekura et al. (2005).

The main observational parameters of these databases are listed in Table 1.

\subsection{Infrared data}

We also investigated the dust distribution using high-resolution (HIRES) IRAS, and MSX data obtained through IPAC ${ }^{1}$. The IR data in the IRAS bands at 60 and $100 \mu \mathrm{m}$ have angular resolutions of $1: 1$ and 1:9. The images in the four MSX bands $(8.3,12.1$, 14.7 , and $21.3 \mu \mathrm{m}$ ) have an angular resolution of 18."3. MSX units were converted into MJy ster ${ }^{-1}$ by multiplying the original figures by $7.133 \times 10^{6}$ (Egan et al. 1999).

\section{Results}

\subsection{Radio continuum emission}

Figure 2 displays the radio continuum image at $843 \mathrm{MHz}$. The image reveals a radio source of $15^{\prime}$ in size, coincident in position with the H II region. There is a remarkable correlation between radio and optical emission regions. The strongest radio emission region coincides with the brightest section of the optical rim at $l \simeq 286^{\circ} 10^{\prime}$. The region of diffuse emission near $(l, b)=$ $\left(286^{\circ} 20^{\prime},-0^{\circ} 4^{\prime}\right)$ also has a weak radio counterpart. The fainter optical regions at $(l, b)=\left(286^{\circ} 13^{\prime},-0^{\circ} 15^{\prime}\right)$ and $\left(286^{\circ} 20^{\prime},-0^{\circ} 7^{\prime}\right)$ also correlate with weak radio emission. Along with the optical image, this radio image shows that the $\mathrm{H}$ II region is far from being homogeneous and that it is non-uniform in density. Gum 31 is also detected in the surveys at 4.85 and $2.4 \mathrm{GHz}$ as an isolated radio source. The flux densities at 4.85 and $2.42 \mathrm{GHz}$ are

${ }^{1}$ IPAC is funded by NASA as part of the IRAS extended mission under contract to Jet Propulsion Laboratory (JPL) and California Institute of Technology (Caltech). 


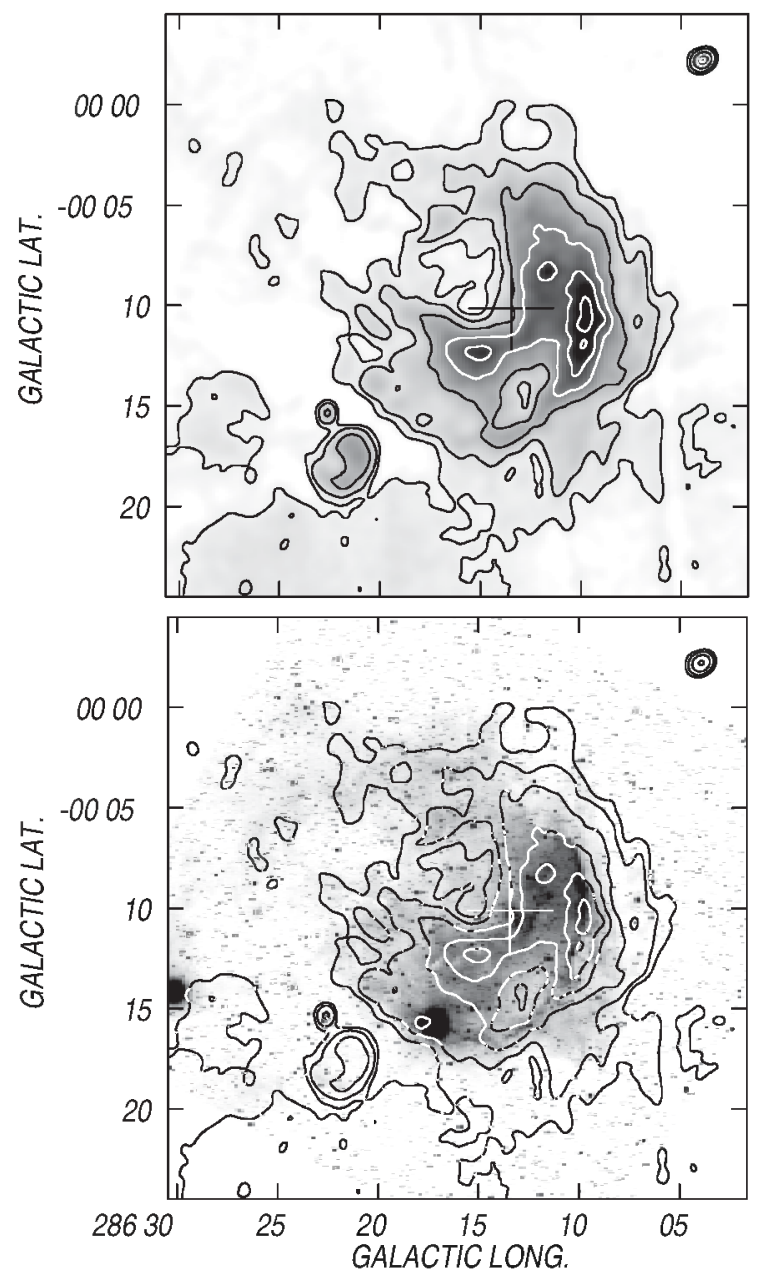

Fig. 2. Top: radio continuum image at $843 \mathrm{MHz}$. The grayscale is from -5 to $180 \mathrm{mJy}$ beam $^{-1}$. The contour lines are $5(5 \sigma), 20,50,100,150$, and $200 \mathrm{mJy}$ beam $^{-1}$. The cross indicates the position of HD 92206 . Bottom: overlay of the image at $843 \mathrm{MHz}$ (contour lines) and the SuperCOSMOS image of Gum 31 (grayscale).

$37.7 \pm 2.5 \mathrm{Jy}$ (coincident with the previous estimate by Caswell \& Haynes [1987D and $40.7 \pm 2.9 \mathrm{Jy}$, respectively.

The spectral index of the radio source, as derived from the emissions at 2.4 and $4.85 \mathrm{GHz}$, is $\alpha=-0.09 \pm 0.20$, confirming its thermal nature. This result is compatible with the detection of RRL at $5 \mathrm{GHz}$.

\subsection{Hi results}

Circular galactic rotation models predict negative radial velocities of up to about $-12 \mathrm{~km} \mathrm{~s}^{-1}$ in the line of sight towards $l$ $=286^{\circ}$. However, radial velocities observed in this section of the Galaxy are more negative (of up to $-30 \mathrm{~km} \mathrm{~s}^{-1}$, Brand \& Blitz 1993), indicating the presence of non-circular motions. Consequently, we paid special attention to the neutral gas emission distribution at negative radial velocities of up to $-50 \mathrm{~km} \mathrm{~s}^{-1}$.

Figure 3 displays a series of $\mathrm{H}$ I images within the velocity interval from -12.8 to $-32.5 \mathrm{~km} \mathrm{~s}^{-1}$ in steps of $3.3 \mathrm{~km} \mathrm{~s}^{-1}$. The presence of a region of low $\mathrm{H}$ I emission surrounded by regions of enhanced emission approximately centered at the position of HD 92206 is clearly identified within the velocity range -12.8 to $-32.5 \mathrm{~km} \mathrm{~s}^{-1}$. An almost complete envelope encircles the void.

The top panel of Fig. 4 displays the H I emission distribution within the velocity range -29.3 to $-16.5 \mathrm{~km} \mathrm{~s}^{-1}$, where the relatively thick envelope, of about $8^{\prime}-10^{\prime}$ (or $7.0-8.7$ pc at $d=$ $3 \mathrm{kpc}$ ), is clearly defined. Within this velocity range, the envelope is more easily identified at higher negative velocities, where the approaching part of the shell is detected.

The bottom panel of Fig. 4 displays an overlay of the $\mathrm{HI}_{\mathrm{I}}$ emission distribution and the SuperCOSMOS image of Gum 31. The H I envelope clearly anti-correlates with the ionized nebula. This envelope is less bright towards higher galactic latitudes.

The systemic velocity of the structure, which corresponds to the velocity at which the feature presents its largest dimensions and deepest temperature gradient, is about $-23 \mathrm{~km} \mathrm{~s}^{-1}$. This velocity is similar to the velocity of the ionized gas $\left(-18 \mathrm{~km} \mathrm{~s}^{-1}\right.$, see Sect. 1) as obtained from RRLs, within the errors.

The morphological agreement between the optical nebula and the H I shell and the agreement in velocity between the ionized and neutral materials indicate that the $\mathrm{H}$ I feature is the neutral counterpart of the ionized nebula.

\subsection{The $\mathrm{CO}$ emission distribution}

The distribution of the molecular gas is shown in Fig. 5. The upper panel displays the ${ }^{12} \mathrm{CO}(1-0)$ emission distribution in grayscale and contour lines, while the bottom panel displays an overlay of the CO contour lines and the optical image of Gum 31. The CO gas distribution displayed in the figure was obtained from the molecular data cube kindly provided by $Y$. Yonekura. We integrated the ${ }^{12} \mathrm{CO}(1-0)$ emission within the velocity interval -27.2 to $-14.0 \mathrm{~km} \mathrm{~s}^{-1}$. This velocity range is slightly different from that used by Yonekura et al. (2005) $(-30$ to $-10 \mathrm{~km} \mathrm{~s}^{-1}$ ), since no CO emission was detected for velocities $v<-27 \mathrm{~km} \mathrm{~s}^{-1}$, and $v>-14 \mathrm{~km} \mathrm{~s}^{-1}$ associated with Gum 31 . Both images, that by Yonekura et al. and the one in Fig. 5, are essentially the same.

Intense $\mathrm{CO}$ emission regions encircle the brightest sections of the optical nebula with CO clumps strikingly bordering the bright rim at $\left(286^{\circ} 10^{\prime},-0^{\circ} 10^{\prime}\right)$, and near $\left(286^{\circ} 23^{\prime},-0^{\circ} 15^{\prime}\right)$, where the nebula appears diffuse. The $\mathrm{CO}$ envelope is open towards $(l, b)=\left(286^{\circ} 25^{\prime},-0^{\circ} 5^{\prime}\right)$. The region of relatively faint optical emission near $(l, b)=\left(286^{\circ} 20^{\prime},-0^{\circ} 4^{\prime}\right)$ coincides with a low CO emission region.

The close morphological agreement between the optical and the molecular emissions in the brightest optical region indicates that the molecular material is interacting with Gum 31 .

The thick lines in the bottom panel of Fig. 5 delineate the $\mathrm{C}^{18} \mathrm{O}$ cores found by Yonekura et al. (2005). The dense cores coincide with the the brightest ${ }^{12} \mathrm{CO}$ emission regions.

The comparison of the molecular gas distribution as shown by the ${ }^{12} \mathrm{CO}$ emission (Fig. 5) with the H I structure (Fig. 4) shows that the neutral envelope around the HII region has a molecular component. Although, both the H I and the molecular envelopes are approximately coincident, most of the bright molecular clumps anticorrelate with the H I maxima, indicating that gas in these regions is mainly in molecular form. However, the comparison of the $\mathrm{CO}$ distribution with the $\mathrm{HI}$ emission distribution at different velocities shows that the ${ }^{12} \mathrm{CO}$ clump at $(l, b)=\left(286^{\circ} 23^{\prime},-0^{\circ} 15^{\prime}\right)$ coincides with an H I cloud present in the velocity interval from -26 to $-29.3 \mathrm{~km} \mathrm{~s}^{-1}$. A possible explanation is the presence of the CO clump inside a dense H I cloud.

The comparison between the ionized, neutral atomic, and molecular distributions around Gum 31 suggests the presence of a prominent PDR bordering the brightest ionized regions. 

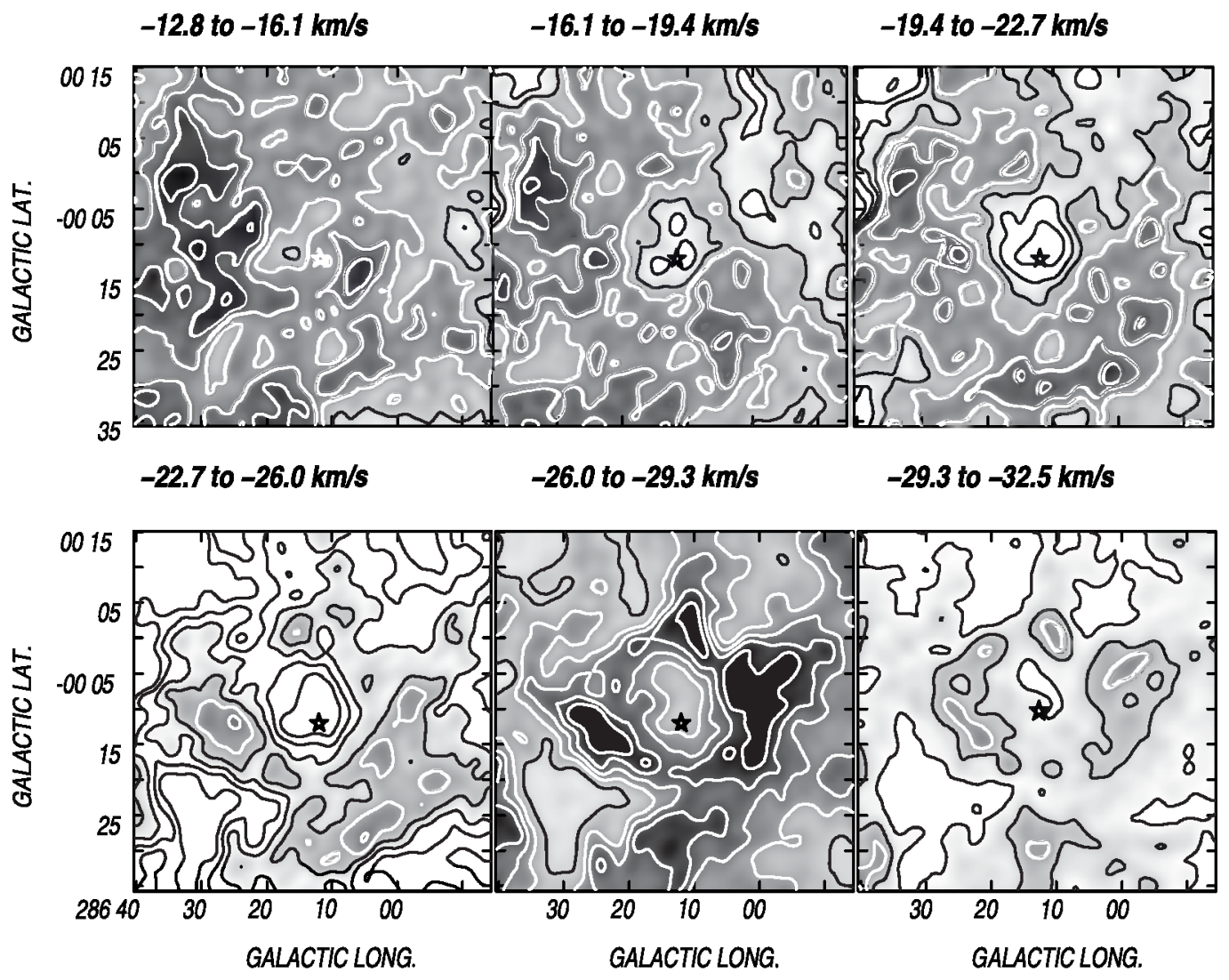

Fig. 3. Series of $\mathrm{HI}$ brightness temperature images for the velocity interval -12.8 to $-32.5 \mathrm{~km} \mathrm{~s}^{-1}$ averaged over $3.3 \mathrm{~km} \mathrm{~s}{ }^{-1}$. The greyscale is from 70 to $120 \mathrm{~K}$ for the images at $v>-26.0 \mathrm{~km} \mathrm{~s}^{-1}$, and from 10 to $60 \mathrm{~K}$ for the images at $v<-26.0 \mathrm{~km} \mathrm{~s}^{-1}$. The contour lines are from 10 to 110 in steps of $10 \mathrm{~K}$. The star indicates the position of HD 92206 . The velocity interval corresponding to each image is indicated.

\subsection{The emission in the infrared}

The distribution of the IR emission at 60 and $100 \mu \mathrm{m}$, due to thermal dust emission, is displayed in Fig. 6. The upper panels show the emission at both wavelengths while the bottom panels display overlays of the IR emission and the optical image of Gum 31. The images reveal an IR structure which is brighter near $(l, b)=\left(286^{\circ} 23^{\prime},-0^{\circ} 15^{\prime}\right)$ and where the optical nebula has its sharpest border, and is weaker on the opposite side. The brightest IR emission regions at $60 \mu \mathrm{m}$ and $100 \mu \mathrm{m}$ are projected onto the neutral envelope, delineating the ionized nebula. The IR emission at $\left(286^{\circ} 23^{\prime},-0^{\circ} 15^{\prime}\right)$ detected at both wavelengths coincides with strong ${ }^{12} \mathrm{CO}(1-0)$ emission. ${ }^{12} \mathrm{CO}$ emission also appears bordering the two IR clumps located near the bright rim at $(l, b)=\left(286^{\circ} 10^{\prime},-0^{\circ} 10^{\prime}\right)$ detected at $60 \mu \mathrm{m}$, and in between. The distribution of the IR emission at both wavelengths shows the presence of dust most probably related to the surrounding H I and molecular shells.

Following the procedure described by Cichowolski et al. (2001), we derived the color temperature of the dust associated with the HII region and the neutral envelope based on the IR fluxes at $60 \mu \mathrm{m}$ and $100 \mu \mathrm{m}$. Taking into account different values for the background emission, we found $T_{\mathrm{d}}=34 \pm 7 \mathrm{~K}$. The range of temperatures corresponds to $n=1-2$ and to different IR background emissions. The parameter $n$ is related to the dust absorption efficiency $\left(\kappa_{r} \propto v^{\prime \prime}\right)$. We adopted $\kappa_{r}=40 \mathrm{~cm}^{2} \mathrm{~g}^{-1}$. This value was derived from the expressions by Hildebrand (1983) for $n=1$ and $\lambda=60 \mu \mathrm{m}$. The dust temperature obtained is typical for $\mathrm{H}$ II regions.

Figure 7 shows an overlay of the distribution of the emission in the MSX bands A and E, and the optical image. The emission in band A closely follows the brightest sections of the nebula, and correlates with the neutral gas. Particularly, the brightest regions emitting at $8.3 \mu \mathrm{m}$ coincide with the dense cores 2 and 6 (see Fig. 5) found by Yonekura et al. (2005). On the contrary, the emission in band $\mathrm{E}$ appears clearly associated with the ionized gas. Note that the strongest emission region in band $\mathrm{A}$ at $(l, b)=\left(286^{\circ} 23^{\prime},-0^{\circ} 15^{\prime}\right)$ coincides with bright molecular and far infrared clumps.

The emission distribution in band $\mathrm{A}$ is most probably related to emission from polycyclic aromatic hydrocarbons (PAHs). According to Cesarsky et al. (1996), these dust grains cannot survive inside the $\mathrm{H}$ II region, but can on the neutral PDR, where they radiate in the PAH bands at 7.7 and $8.6 \mu \mathrm{m}$, included in the MSX band A. MSX band E, on the contrary, includes continuum emission from very small grains, which can survive inside ionized regions (cf. Deharveng et al. 2005), and a contribution from nebular emission lines.

In summary, the distribution of the neutral atomic and molecular gas, and that of the interstellar dust reveals the presence of a neutral shell surrounding the HII region. The distribution of the molecular gas and that of the emission in the MSX band A related to PAHs strongly suggests the presence of a PDR at the interface between the ionized and the molecular gas.

Both the infrared emission at 60 and $100 \mu \mathrm{m}$ and the optical image suggest that the faint optical emission region near $(l, b)=\left(286^{\circ} 20^{\prime},-0^{\circ} 4^{\prime}\right)$ (indicated by an arrow in the bottom right image of Fig. 6) are also linked to Gum 31. As pointed out in Sect. 3.1, this region is also faint in the radio continuum (see Fig. 2), and corresponds to weak regions in the $\mathrm{HI}$ and $\mathrm{CO}$ envelopes suggesting that the ionizing flux of the massive stars in 

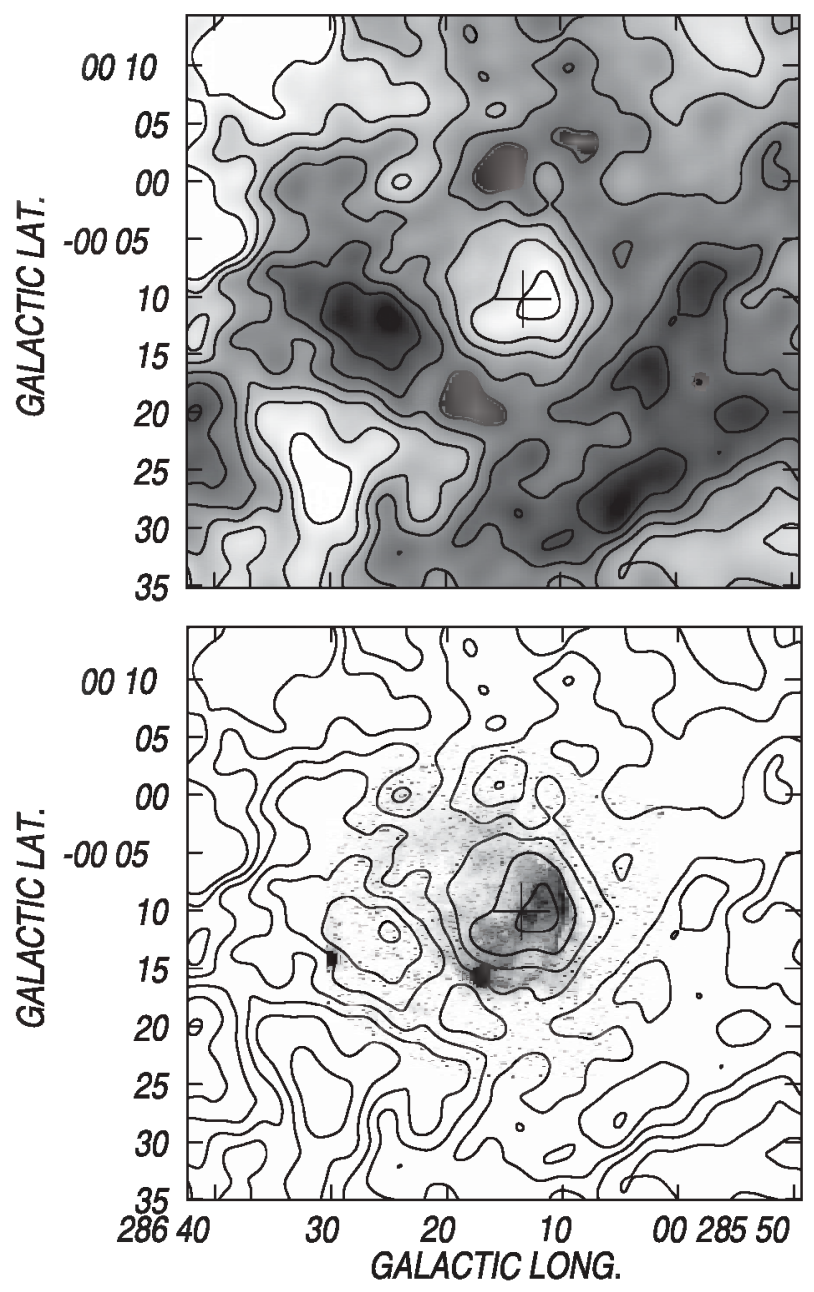

Fig. 4. Top: H I brightness temperature image corresponding to the velocity interval -29.3 to $-16.5 \mathrm{~km} \mathrm{~s}^{-1}$ showing the $\mathrm{H} \mathrm{I} \mathrm{structure} \mathrm{related} \mathrm{to}$ Gum 31 . The grayscale is from 40 to $100 \mathrm{~K}$. The contour lines are from 40 to $100 \mathrm{~K}$ in steps of $10 \mathrm{~K}$. Bottom: overlay of the SuperCOSMOS (grayscale) and HI (contours) images showing the close correspondence of neutral and ionized gas emissions.

the open cluster may drain through these regions to the general interstellar medium. The situation resembles the case of the stellar wind bubble around WR 23 (Cappa et al. 2005).

\section{Discussion}

\subsection{Physical parameters}

The main parameters of the dust and the ionized and neutral gas related to Gum 31 are summarized in Table 2. The parameters of the ionized gas were derived from the image at $4.85 \mathrm{GHz}$. The uncertainty in the flux density corresponds to an error of $0.1 \mathrm{Jy} \mathrm{beam}^{-1}$ in the estimate of the radio continulum background. The electron density and the H II mass were obtained from the expressions by Mezger \& Henderson (1967) for a spherical H II region of constant electron density (rms electron density $n_{\mathrm{e}}$ ). The presence of He II was considered by multiplying the H I mass by 1.27. The number of UV photons necessary to ionize the gas $\log N_{\mathrm{Ly}-\mathrm{c}}$ was derived from the radio continuum results. Errors in the linear radius, in the rms electron density, and in the excitation parameter come from the distance uncertainty. The high electron density of the $\mathrm{H}$ II region is compatible with the relatively short lifetime for the cluster.
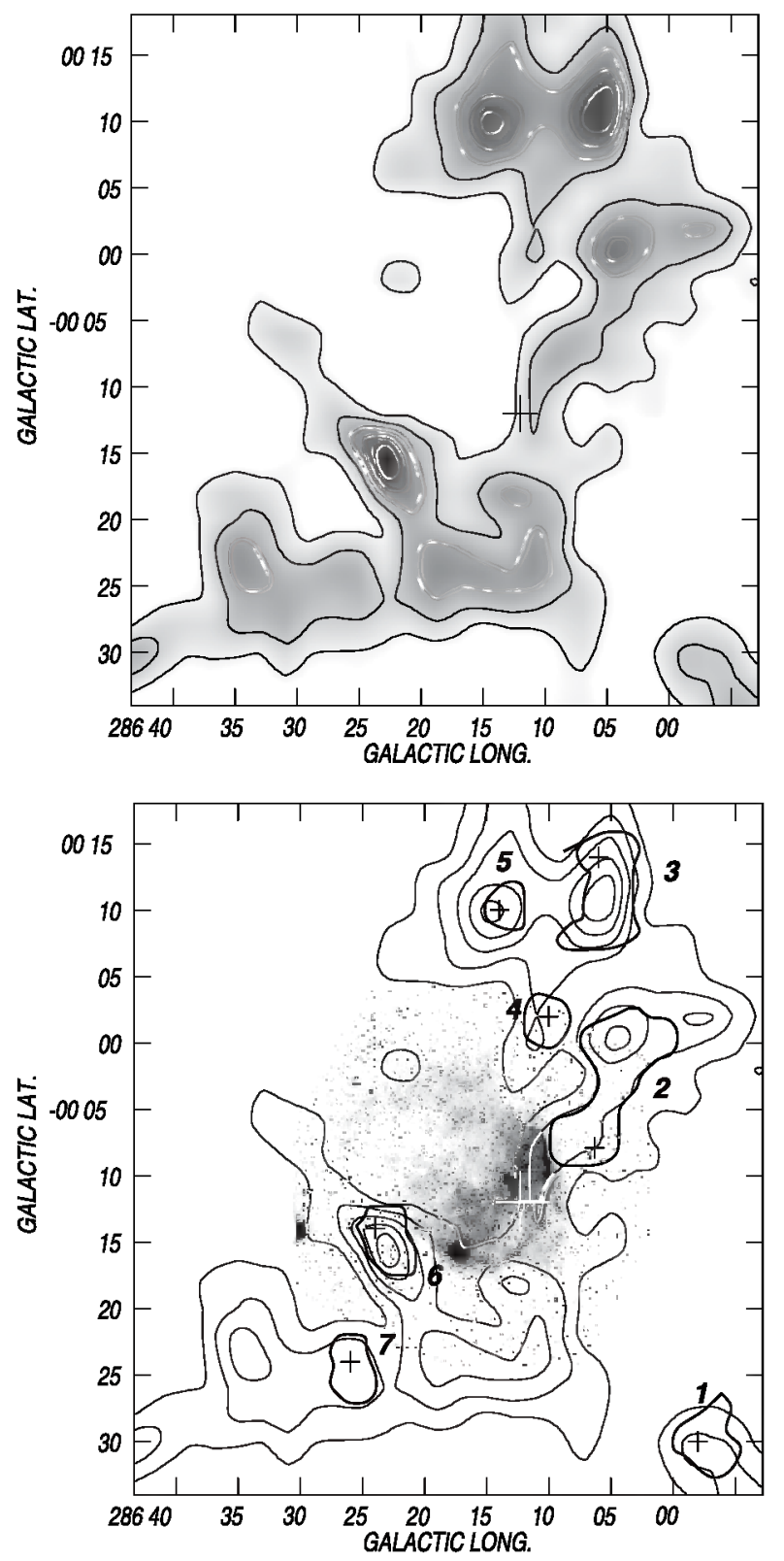

Fig. 5. Top: ${ }^{12} \mathrm{CO}(1-0)$ emission distribution corresponding to the velocity interval -27.2 to $-14.0 \mathrm{~km} \mathrm{~s}^{-1}$. The grayscale is from 15 to $150 \mathrm{~K} \mathrm{~km} \mathrm{~s}^{-1}$. The contour lines are 20.2,40.5, 60.7, 81.0 and $101.2 \mathrm{~K} \mathrm{~km} \mathrm{~s}^{-1}$. Bottom: overlay of the SuperCOSMOS (grayscale) and the $\mathrm{CO}$ (contours) images. The thick lines delineate the $\mathrm{C}^{18} \mathrm{O}$ cores described by Yonekura et al. (2005). The numbers correspond to the $\mathrm{C}^{18} \mathrm{O}$ cores as identified in Table 2 by Yonekura et al., and the crosses mark the core positions indicated in the same table.

The parameters of the neutral atomic gas includes: the $(l, b)$ position of the centroid of the HI shell, the velocity interval spanned by the structure, the systemic and expansion velocities, the radius of the neutral gas structure, and the associated atomic mass.

The expansion velocity was estimated as in previous papers (see Cappa et al. 2005 and references therein) as $v_{\exp }=\left(v_{2}-\right.$ $\left.v_{1}\right) / 2+1.6 \mathrm{~km} \mathrm{~s}^{-1}$. The extra $1.6 \mathrm{~km} \mathrm{~s}^{-1}$ allows for the presence of $\mathrm{H} \mathrm{I}$ in the caps of the expanding shell, which should be present in the images at $v_{1}+1.6 \mathrm{~km} \mathrm{~s}^{-1}$ and $v_{2}-1.6 \mathrm{~km} \mathrm{~s}^{-1}$. These caps are not detected in the present case, as in most of the cases, probably because of their weak brightness temperature. 

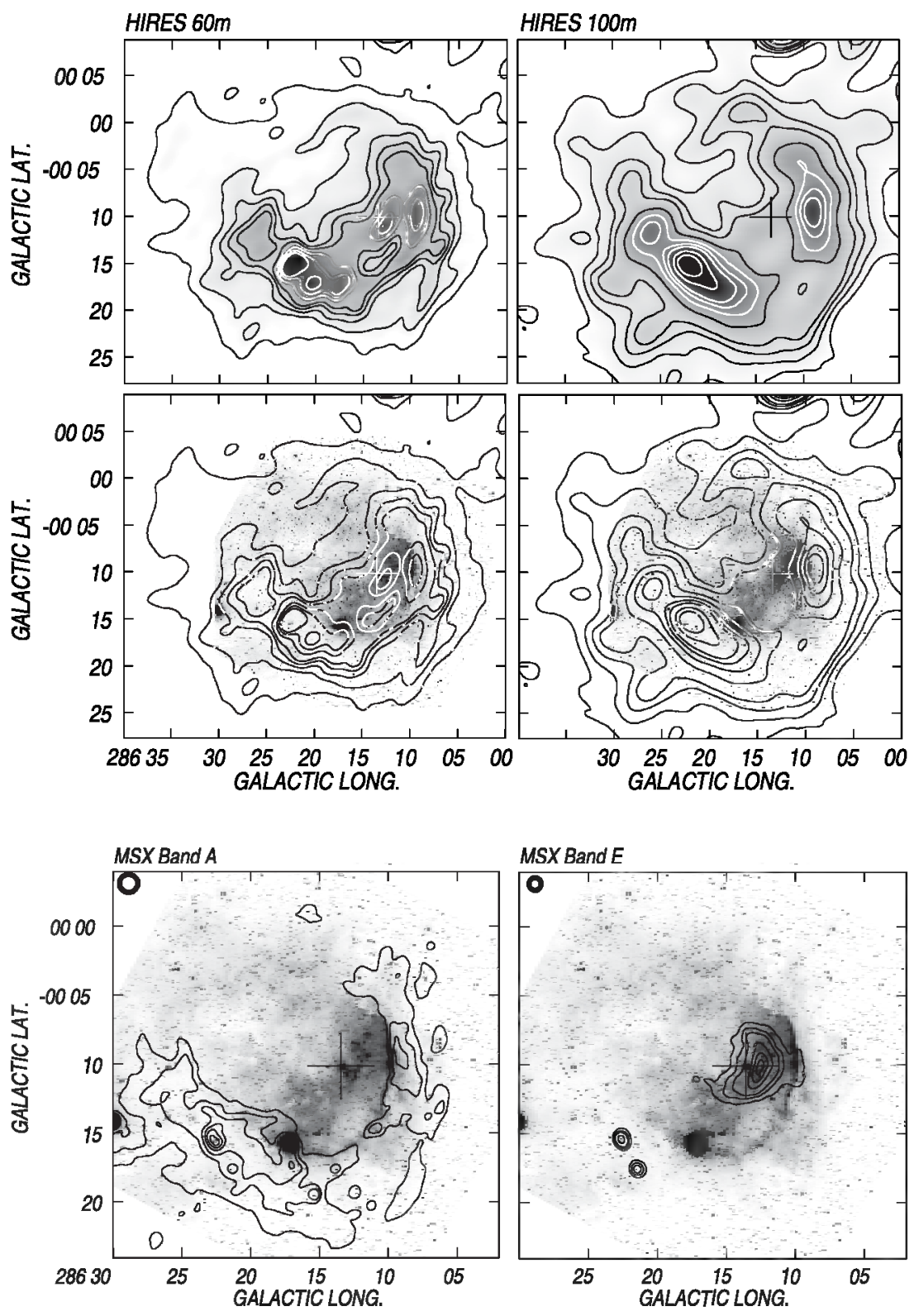

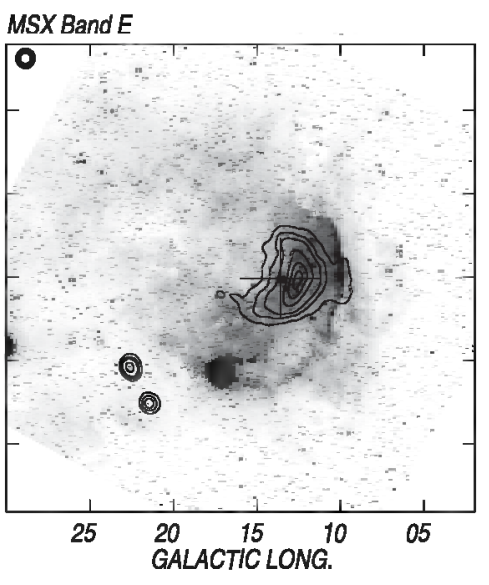

Fig. 6. Top: far infrared IRAS images at 60 and $100 \mu \mathrm{m}$ towards Gum 31. The grayscale is from 300 to $3000 \mathrm{MJy} \mathrm{ster}^{-1}$ for the image at $60 \mu \mathrm{m}$. and from 600 to $4500 \mathrm{MJy} \mathrm{ster}^{-1}$ for the image at $100 \mu \mathrm{m}$. The contour lines are from 200 to $1000 \mathrm{MJy} \mathrm{ster}^{-1}$ in steps of $200 \mathrm{MJy} \mathrm{ster}^{-1}$ and from 1000 to $3000 \mathrm{MJy} \mathrm{ster}^{-1}$ in steps of $500 \mathrm{MJy} \mathrm{ster}^{-1}$ for the image at $60 \mu \mathrm{m}$. and from 400 to $1000 \mathrm{MJy} \mathrm{ster}^{-1}$ in steps of $200 \mathrm{MJy} \mathrm{ster}^{-1}$ and from 1000 to $3000 \mathrm{MJy}^{-1}$ ster $^{-1}$ in steps of $500 \mathrm{MJy} \mathrm{ster}^{-1}$ for the image at $100 \mu \mathrm{m}$. Bottom: overlay of the optical (SuperCOSMOS) (grasscale) and the IR images.
Fig. 7. Overlay of the MSX infrared images (contours) corresponding to bands $\mathrm{A}(8.28 \mu \mathrm{m})$ and $\mathrm{E}$ $(21.34 \mu \mathrm{m})$. and the SuperCOSMOS image of the nebula (grayscale). The contour lines are 25, 39, 57. 85 . 114. and $140 \mathrm{MJy} \mathrm{ster}^{-1}$ for band A; and 36. 46. 57. 85 . 114. and $140 \mathrm{MJy} \mathrm{ster}^{-1}$ for band $E$.
The radius of the $\mathrm{HI}$ structure was estimated from Fig. 4 and corresponds to the position of the maxima in the shell. The neutral atomic mass corresponds to the mass excess in the shell, assuming that the gas is optically thin and including a He abundance of $10 \%$.

The $\mathrm{H}_{2}$ column density $\left(N_{\mathrm{H} 2}\right)$ and the molecular mass were estimated from the ${ }^{12} \mathrm{CO}$ data, making use of the empirical relation between the integrated emission $W_{\mathrm{CO}}\left(=\int T \mathrm{~d} v\right)$ and $N_{\mathrm{H} 2}$. We adopted $N_{\mathrm{H} 2}=(1.06 \pm 0.14) \times \mathrm{W}_{\mathrm{CO}} \times 10^{20} \mathrm{~cm}^{-2}$ $\left(\mathrm{K} \mathrm{km} \mathrm{s}^{-1}\right)^{-1}$, obtained from $\gamma$-ray studies of molecular clouds in the Orion region (Digel et al. 1995). To derive the molecular mass, we integrated the $\mathrm{CO}$ emission within a circle of about $16^{\prime}$ in radius $(=15 \mathrm{pc}$ at $3.0 \mathrm{kpc})$, centered at $(l, b)=\left(286^{\circ} 16^{\prime}\right.$, $\left.-0^{\circ} 8^{\prime}\right)$.

The ambient density derived by distributing the molecular mass over a sphere of $15.0 \mathrm{pc}$ in radius is $\simeq 350 \mathrm{~cm}^{-3}$, reinforcing the idea that the molecular gas represents the remains of the original material where the open cluster NGC 3324 was born. The difference between the electron density and the ambient density indicates that the H II region is expanding.
It is known that massive stars have stellar winds with high mass loss rates and terminal velocities (Chlebowski \& Garmany 1991; Prinja et al. 1990), which generally affect the surrounding interstellar medium creating interstellar bubbles (Weaver et al. 1977). In the optical domain, they appear as ring-shaped structures (Lozinskaya 1982). The optical appearance of the H II region, almost spherical without clear evidence for a central cavity, suggests that the massive stars in the cluster have weak stellar winds (which is supported by the short age of the cluster) and/or have existed during a very short period of time to create an interstellar bubble in an interstellar medium as dense as observed here.

Figure 8 shows schematically the distribution of the different gas components. Although H I and molecular clumps anticorrelate in position, the large scale H I and molecular gas coincide in position around the nebula but in the region at higher galactic latitudes, where the $\mathrm{CO}$ emission is observed outside the H I region. The large amount of molecular gas compared to the neutral atomic gas supports the idea that the $\mathrm{CO}$ emission which encompasses the ionized nebula represents the remains of the molecular 
Table 2. Main parameters of the ionized and neutral gas in Gum 31.

\begin{tabular}{|c|c|}
\hline Adopted distance $(\mathrm{kpc})$ & $3.0 \pm 0.5$ \\
\hline \multicolumn{2}{|l|}{ H II region } \\
\hline Flux density at $4.85 \mathrm{GHz}(\mathrm{Jy})$ & $37.7 \pm 2.5$ \\
\hline Angular radius (arcmin) & $7.5 \pm 0.2$ \\
\hline Linear radius (pc) & $6.5 \pm 1.0$ \\
\hline Emission measure $\left(\mathrm{pc} \mathrm{cm}^{-6}\right)$ & $(1.5 \pm 0.2) \times 10^{4}$ \\
\hline rms electron density $\left[n_{\mathrm{e}}\left[\left(\mathrm{cm}^{-3}\right)\right.\right.$ & $33 \pm 3$ \\
\hline Used Lyman UV photons $\left[\log N_{\mathrm{Ly}-\mathrm{c}}\left[\left(\mathrm{s}^{-1}\right)\right.\right.$ & $49.0 \pm 0.1$ \\
\hline Ionized mass $\left(M_{\odot}\right)$ & $3300 \pm 1100$ \\
\hline \multicolumn{2}{|l|}{ Neutral atomic shell } \\
\hline$(l, b)$ centroid & $286^{\circ} 15^{\prime},-0^{\circ} 10^{\prime}$ \\
\hline Velocity interval $v_{1}, v_{2}\left(\mathrm{~km} \mathrm{~s}^{-1}\right)$ & $-13,-32$ \\
\hline H I systemic velocity $\left(\mathrm{km} \mathrm{s}^{-1}\right)$ & -23 \\
\hline Expansion velocity $v_{\mathrm{exp}}\left(\mathrm{km} \mathrm{s}^{-1}\right)$ & 11 \\
\hline Radius of the H I structure (arcmin) & 11.5 \\
\hline Radius of the H I structure $R(\mathrm{pc}$ ) & $10.0 \pm 1.7$ \\
\hline Atomic mass in the shell $\left(M_{\odot}\right)$ & $1500 \pm 500$ \\
\hline \multicolumn{2}{|l|}{ Molecular shell } \\
\hline Velocity interval $v_{1}, v_{2}\left(\mathrm{~km} \mathrm{~s}^{-1}\right)$ & $-27.2,-14.0$ \\
\hline Mean $\mathrm{H}_{2}$ column density $\left(\mathrm{cm}^{-2}\right)$ & $1.2 \times 10^{22}$ \\
\hline Molecular mass $\left(M_{\odot}\right)$ & $(1.1 \pm 0.5) \times 10^{5}$ \\
\hline \multicolumn{2}{|l|}{ Dust related to the $H \amalg$ region and the neutral shell } \\
\hline Total dust mass $\left(M_{\odot}\right)$ & $60 \pm 20$ \\
\hline Dust color temperature $(\mathrm{K})$ & $34 \pm 7$ \\
\hline
\end{tabular}

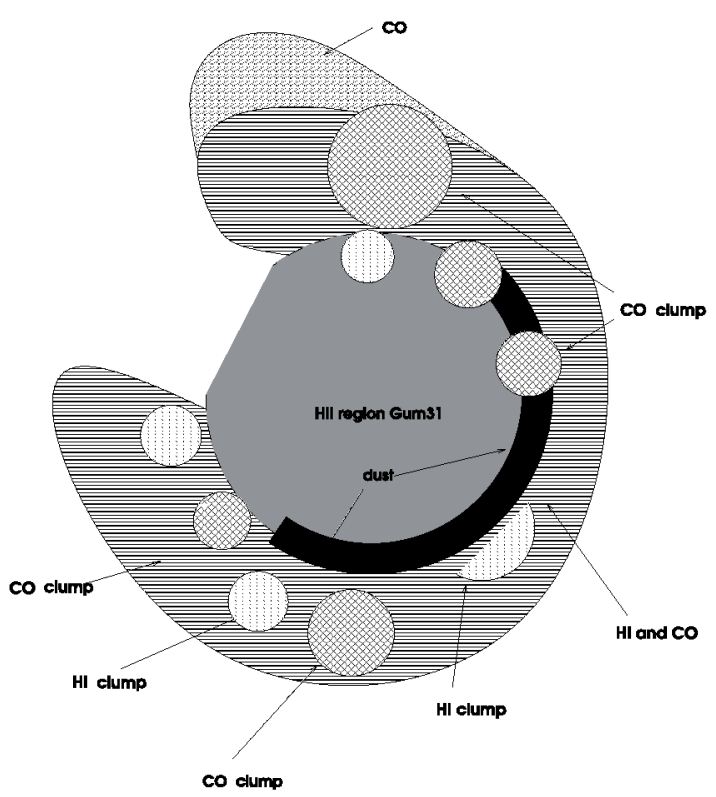

Fig. 8. Schematic view of Gum 31 and the respective locations of the different gas components and of interstellar dust.

material where the open cluster was born. Part of this H I gas may have originated in the photodissociation of the molecular gas.

\subsection{Energy budget}

Bearing in mind that massive stars have a copious UV flux capable of ionizing the surrounding $\mathrm{H}$ I gas, we investigate in this section whether the massive stars in NGC 3324 can provide the energy to ionize the gas.

As described in Sect. 1, the only O-type stars in the open cluster are the multiple system HD 92206, which contains three O-type stars classified as O6.5V, O6.5V and O8.5 (Walborn 1982; Mathys 1988). Considering that HD 92206A is almost
1 mag brighter than component B (Clariá 1977), the similar spectral types found by Mathys (1988) suggest that HD 92206A is probably a spectroscopic binary $06.5 \mathrm{~V}+06.5 \mathrm{~V}$. Taking into account the UV photon fluxes emitted by the stars, $N_{*}\left(\mathrm{~s}^{-1}\right)$, derived by Martins et al. (2005) from stellar atmosphere models, a group of four massive stars having the spectral types indicated above have a total UV photon flux corresponding to $\log N_{*}=49.4$. By comparing $N_{*}$ with the UV photons used to ionize the gas $\left(N_{\mathrm{Ly}-\mathrm{c}}\right)$ listed in Table 2, we conclude that the massive O-type stars in NGC 3324 can maintain the H II region ionized.

The radius of the Strömgren sphere formed in a region with a volumetric ambient density of $350 \mathrm{~cm}^{-3}$ is $\simeq 1.7 \mathrm{pc}$, which is lower than the radius of the ionized region. Following Spitzer (1978), we estimated that the H II region has been expanding for $\approx 1 \times 10^{6} \mathrm{yr}$.

The ambient density we derived from the ${ }^{12} \mathrm{CO}(1-0)$ data is larger than the rms electron density, $33 \mathrm{~cm}^{-3}$ (see Table 2), giving additional support for the interpretation that the HII region is expanding.

\section{Stellar formation}

Stellar formation may be induced by the expansion of the H II region in the surrounding molecular envelope, where the presence of high density regions favors the conditions which lead to the formation of new stars. Among the different processes that induce star formation, the collect and collapse process proposed by Elmegreen \& Lada (1977) may work efficiently in the dense molecular shells around H II regions. The physical conditions in the molecular shells were discussed by different authors (Whitworth et al. 1994; Hosokawa \& Inutsuka 2005, 2006; Dale et al. 2007).

To investigate the presence of protostellar candidates in this region we used data from the IRAS, MSX and 2MASS point source catalogs. We searched for point sources in a region of about $20^{\prime}$ in radius centered at the position of the NGC 3324 . 
Table 3. IR Point sources and YSO candidates from the IRAS, MSX and 2MASS catalogs.

\begin{tabular}{|c|c|c|c|c|c|c|c|c|}
\hline \multirow[t]{2}{*}{ \# } & \multirow[t]{2}{*}{$l\left[0^{\prime}[\right.$} & \multirow[t]{2}{*}{$b\left[o^{\prime}[\right.$} & \multirow[t]{2}{*}{ IRAS source } & \multicolumn{4}{|c|}{ Fluxes [Jy[ } & \multirow[t]{2}{*}{$L_{\mathrm{IRAS}}\left[10^{3} L_{B=}\right]$} \\
\hline & & & & $12 \mu \mathrm{m}$ & $25 \mu \mathrm{m}$ & $60 \mu \mathrm{m}$ & $100 \mu \mathrm{m}$ & \\
\hline 1 & $285: 59: 82$ & $+005: 82$ & $10349-5801$ & 1.6 & 2.5 & 27.9 & 104 & 1.5 \\
\hline 2 & $28604: 98$ & -025.08 & $10335-5830$ & 0.8 & 3.5 & 22.8 & 111 & 1.5 \\
\hline 3 & $286^{\circ} 08^{\prime} 58$ & $-0^{\circ} 06^{\prime} .96$ & $10351-5816$ & 6.3 & 6.8 & 315 & 1480 & 18 \\
\hline 4 & $286^{\circ} 08^{\prime} 64$ & $-018: 78$ & $10343-5826$ & 5.5 & 4.3 & 103 & 340 & 5 \\
\hline 5 & $286^{\circ} 10^{\prime} 92$ & $-0^{\prime} 14^{\prime} 7$ & $10349-5824$ & 5.9 & 9.0 & 163 & 1660 & 18 \\
\hline 6 & $286^{\circ} 12 ! 18$ & $+010: 20$ & $10365-5803$ & 7.2 & 86 & 1170 & 2780 & 43 \\
\hline 7 & $286^{\circ} 15: 12$ & $-0^{\circ} 25^{\prime} .44$ & $10346-5835$ & 1.1 & 3.3 & 11.7 & 1430 & 14 \\
\hline 8 & $286^{\circ} 17: 34$ & $+0^{\prime} 00^{\prime} \cdot 42$ & $10365-5814$ & 2.3 & 1.7 & 85 & 246 & 4 \\
\hline 9 & $28622: 5$ & $-0: 15: 3$ & $10361-5830$ & 12.4 & 38.4 & 626 & 2160 & 30 \\
\hline 10 & $286: 26: 94$ & $-022: 92$ & $10361-5839$ & 2.8 & 5.4 & 51.6 & 307 & 4 \\
\hline 11 & $28629 ! 34$ & $-016: 68$ & $10368-5835$ & 2.1 & 2.3 & 84 & 271 & 4 \\
\hline 12 & $28633: 72$ & $-0^{\circ} 07: 08$ & $10379-5828$ & 1.0 & 2.0 & 13.8 & 540 & 5 \\
\hline \multirow[t]{2}{*}{$\#$} & $l\left[o^{\prime}[\right.$ & $b\left[0^{\prime}[\right.$ & MSX source & \multicolumn{4}{|c|}{ Fluxes [Jy[ } & \\
\hline & & & & $8 \mu \mathrm{m}$ & $12 \mu \mathrm{m}$ & $14 \mu \mathrm{m}$ & $21 \mu \mathrm{m}$ & Class. \\
\hline 13 & $286^{\circ} 09: 78$ & $-0^{\circ} 11: 28$ & G286.1626-00.1877 & 0.7311 & 1.298 & 1.256 & 2.806 & CHII \\
\hline 14 & $286^{\prime} 12 ! 36$ & $-0 \div 09: 66$ & G286.2056-00.1611 & 0.1585 & 0.9313 & 2.151 & 6.904 & MYSO \\
\hline 15 & $286^{\circ} 12: 48$ & $-010: 32$ & G286.2077-00.1720 & 0.0870 & 0.9481 & 1.764 & 2.9 & MYSO \\
\hline 16 & $286^{\prime \prime} 12.54$ & $+0^{\prime} 10^{\prime} \cdot 14$ & G286.2086+00.1694 & 1.353 & 2.882 & 7.182 & 40.57 & MYSO \\
\hline 17 & $286^{\prime} 12: 6$ & $-0^{\prime} 10^{\prime} 68$ & G286.2096-00.1775 & 0.2202 & 0.9538 & 1.368 & 7.206 & MYSO \\
\hline 18 & $286: 15: 42$ & $-0: 19: 44$ & $\mathrm{G} 286.2566-00.3236$ & 2.04 & 2.151 & 1.34 & 4.328 & $\mathrm{CHII}$ \\
\hline 19 & $286: 21: 48$ & $-0.17: 58$ & G286.3579-00.2933 & 0.7126 & 1.815 & 2.677 & 6.065 & MYSO \\
\hline 20 & $286: 22: 5$ & $-0: 15: 78$ & G286.3747-00.2630 & 3.591 & 4.756 & 2.409 & 7.577 & $\mathrm{CH} I I$ \\
\hline 21 & $286: 22: 62$ & $-0: 15: 36$ & G286.3773-00.2563 & 1.628 & 2.918 & 3.855 & 12.1 & MYSO \\
\hline \# & $l\left[\mathrm{o}^{\prime}[\right.$ & $b\left[o^{\prime}[\right.$ & 2MASS source & $J$ [mag [ & $H[\mathrm{mag}[$ & $K_{\mathrm{s}}[\mathrm{mag}[$ & $(J-H)$ & $(H-K)$ \\
\hline 22 & $285^{\circ} 54^{\prime} 42$ & $-013: 74$ & $10350210-5831039$ & 10.584 & 10.631 & 10.564 & -0.047 & 0.067 \\
\hline 23 & $286: 04: 44$ & $-0: 00: 24$ & $10365972-5824186$ & 12.042 & 10.51 & 9.284 & 1.532 & 1.226 \\
\hline 24 & $286^{\circ} 06 ! 36$ & -0.08 .64 & $10364112-5832326$ & 11.367 & 11.385 & 11.28 & -0.018 & 0.105 \\
\hline 25 & $28606: 54$ & $-0.08: 40$ & $10364296-5832267$ & 10.465 & 10.513 & 10.439 & -0.048 & 0.074 \\
\hline 26 & $286^{\circ} 09^{\prime} 60$ & $-0: 00: 30$ & $10373406-5826540$ & 12.487 & 11.454 & 10.513 & 1.033 & 0.941 \\
\hline 27 & $286: 09: 78$ & $-011: 22$ & $10365396-5836293$ & 12.242 & 10.99 & 10.103 & 1.252 & 0.887 \\
\hline 28 & $286^{\prime} 10 ! 08$ & $-009: 12$ & $10370395-5834489$ & 10.772 & 9.712 & 8.685 & 1.06 & 1.027 \\
\hline 29 & $286^{\prime} 10: 20$ & $-0^{\prime} 11 ! 10$ & $10365749-5836366$ & 13.99 & 12.815 & 11.984 & 1.175 & 0.831 \\
\hline 30 & $286^{\circ} 11^{\prime} 16$ & $-0^{\circ} 02^{\prime} .64$ & $10373574-5829405$ & 15.318 & 13.343 & 11.614 & 1.975 & 1.729 \\
\hline 31 & $286^{\circ} 12: 96$ & $-004 ! 86$ & $10373956-5832311$ & 10.926 & 10.752 & 10.554 & 0.174 & 0.198 \\
\hline 32 & $286^{\circ} 13: 02$ & $-010 ! 86$ & $10371717-5837460$ & 11.793 & 11.773 & 11.665 & 0.02 & 0.108 \\
\hline 33 & $286^{\circ} 13: 32$ & $-0^{\circ} 08.46$ & $10372824-5835492$ & 12.191 & 11.301 & 10.448 & 0.89 & 0.853 \\
\hline 34 & $286: 13: 38$ & $-0^{\prime} 10^{\prime} \cdot 20$ & $10372226-5837229$ & 7.563 & 7.588 & 7.479 & -0.025 & 0.109 \\
\hline 35 & $286^{\circ} 13: 92$ & $-0^{\circ} 17: 87$ & $10365763-5844052$ & 12.22 & 11.882 & 11.51 & 0.338 & 0.372 \\
\hline 36 & $286: 19: 26$ & $-0.06: 84$ & $10381421-5837192$ & 12.635 & 11.886 & 11.349 & 0.749 & 0.537 \\
\hline 37 & $28620 ! 10$ & $-0^{\prime} 19: 80$ & $10373105-5849026$ & 15.155 & 12.8 & 11.127 & 2.355 & 1.673 \\
\hline 38 & 28621.06 & $-016 ! 86$ & $10375219-5847133$ & 12.271 & 11.363 & 10.675 & 0.908 & 0.688 \\
\hline 39 & $28621: 90$ & $-004: 92$ & $10383875-5836566$ & 12.116 & 11.636 & 11.187 & 0.48 & 0.449 \\
\hline 40 & $28622 ! 92$ & $-013: 20$ & $10381461-5844416$ & 12.367 & 11.815 & 11.398 & 0.552 & 0.417 \\
\hline 41 & 28624.92 & -018.66 & $10380736-5850240$ & 12.477 & 11.538 & 10.78 & 0.939 & 0.758 \\
\hline 42 & $286: 28: 11$ & $-024: 50$ & $10380702-5857039$ & 12.724 & 11.599 & 10.726 & 1.125 & 0.873 \\
\hline 43 & 28628.44 & $-023: 71$ & $10381226-5856318$ & 13.348 & 12.274 & 11.355 & 1.074 & 0.919 \\
\hline 44 & $286: 29: 46$ & -0.06 .30 & $10392451-5841486$ & 13.836 & 12.561 & 11.619 & 1.275 & 0.942 \\
\hline 45 & $28629: 46$ & $-0^{\circ} 08^{\prime} .10$ & $10391799-5843257$ & 13.195 & 12.322 & 11.624 & 0.873 & 0.698 \\
\hline 46 & $28630 ! 90$ & $-0.06: 30$ & $10393410-5842321$ & 11.701 & 10.423 & 9.334 & 1.278 & 1.089 \\
\hline 47 & $28631: 27$ & -028.40 & $10381363-5902003$ & 13.368 & 12.557 & 11.982 & 0.811 & 0.575 \\
\hline
\end{tabular}

\subsection{IRAS sources}

The IRAS point source catalog allows identification of protostellar candidates following the criteria by Junkes et al. (1992). Sources with quality factors $Q_{60}+Q_{100} \geq 4$ were considered. Twelve out of the thirteen point sources have IR spectra compatible with protostellar objects. The names of the IRAS protostellar candidates, their $(l, b)$ position, their fluxes at different IR wavelengths, and their luminosity derived following Yamaguchi et al. (2001), along with a reference number are listed in Table 3. The IRAS protostellar candidates are indicated as filled triangles in Fig. 9, superimposed onto the molecular gas and ionized gas distributions. Each source can be identified in the figure by its reference number. They are probably young stellar objects (YSOs).

\subsection{MSX sources}

Massive young stellar objects (MYSOs) can be identified from the MSX point catalogue following the criteria by Lumsden et al. (2002). A total of 310 MSX point sources were found to be projected onto the area.

Lumsden et al. (2002) found that MYSOs have infrared fluxes with ratios $F_{21} / F_{8}>2$ and $F_{14} / F_{12}>1$, where $F_{8}$, 


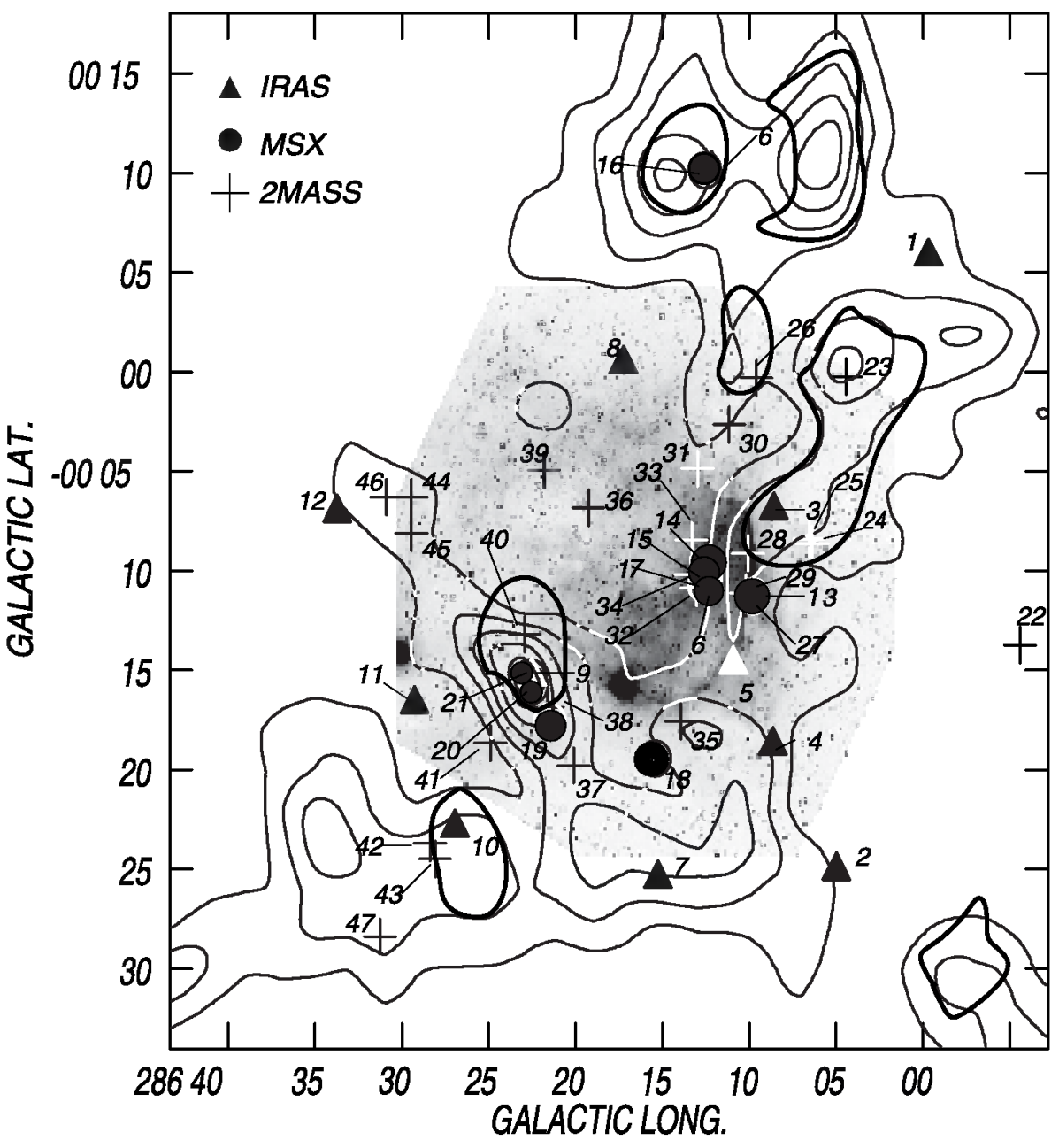

Fig.9. Top: point sources from the IRAS (triangles), MSX (circles), and 2MASS catalogs overlaid onto the SuperCOSMOS image and the ${ }^{12} \mathrm{CO}$ and $\mathrm{C}^{18} \mathrm{O}$ contours (see Fig. 5 for details).
$F_{12}, F_{14}$, and $F_{21}$ are the fluxes at $8.3,12,14$, and $21 \mu \mathrm{m}$. For compact $\mathrm{H}$ II regions, ratios are $F_{21} / F_{8}>2$ and $F_{14} / F_{12}<1$. Taking into account sources with flux quality $q \geq 2$, we were left with 14 sources after applying Lumsden et al.'s criteria indicated above.

Six out of the 14 sources can be classified as MYSOs, while three sources are compact H II regions, also indicative of active stellar formation. The nine sources are listed in Table 3 and indicated in Fig. 9 as circles.

\subsection{MASS sources}

Point sources with infrared excess, which are YSO candidates, were searched for in the 2MASS catalog (Cutri et al. 2003), which provides detections in three near IR bands: $J, H$, and $K_{\mathrm{S}}$, at $1.25,1.65$, and $2.17 \mu \mathrm{m}$, respectively. A total of $2 \times$ $10^{4}$ sources are projected onto a circular region of $20^{\prime}$ in radius. We took into account sources with $S / N>10$ (corresponding to quality "AAA"). Only sources with $K_{\mathrm{S}}<12$ were included. The last criterium corresponds to stars with spectral types earlier than B3 at a distance of $3.0 \mathrm{kpc}$. Following Comerón et al. (2005) and Romero (2006), we determined the parameter $q=$ $(J-H)-1.83 \times\left(H-K_{\mathrm{s}}\right)$. Sources with $q \leq-0.15$ are classified as objects with infrared excess which may be YSOs. After applying this criterium we were left with 26 sources, which are shown in the color-color and magnitude-color diagrams of Fig. 10. Absolute magnitudes for the ZAMS in the $K_{\mathrm{S}}$ band are from Hanson et al. (1997), while values corresponding to $(J-H)$ and $\left(H-K_{\mathrm{S}}\right)$ colors were obtained from Koornneef (1983). The location of these sources is also marked in Fig. 9 as crosses. The main data of these sources are shown in Table 3 .

The selected sources are located to the right of the reddening vector in the color-color diagram, where YSOs are expected to be placed. Most of the sources with the highest infrared excess appear projected onto the molecular clouds (see Fig. 9). The magnitude-color diagram shows that most of the sources are MYSOs. Although these diagrams are not conclusive in identifying YSOs, the strong infrared excess of the sources is compatible with protostellar candidates.

\subsection{Distribution and characteristics of the YSOs}

Figure 9 shows that the IRAS and MSX point sources, and most of the 2MASS point sources classified as YSOs appear bordering the ionized region, projected onto the molecular envelope detected in ${ }^{12} \mathrm{CO}$ emission, close to the periphery of the $\mathrm{H}$ II region, or near the central cluster. Some are also coincident with the dense cores found by Yonekura et al. (2005) in $\mathrm{C}^{18} \mathrm{O}$. We analyze some particular regions.

The IRAS source \#9, the MSX sources \#19, \#20, and \#21, and the 2MASS sources \#38 and \#40, are projected onto a ${ }^{12} \mathrm{CO}$ clump and onto the dense core 6 found in $\mathrm{C}^{18} \mathrm{O}$. The presence of these sources indicates that stellar formation is ongoing in this particular molecular clump. As suggested by Yonekura et al., a star cluster including massive stars is probably being formed in this region. The MSX source \#19 is coincident with an extended 

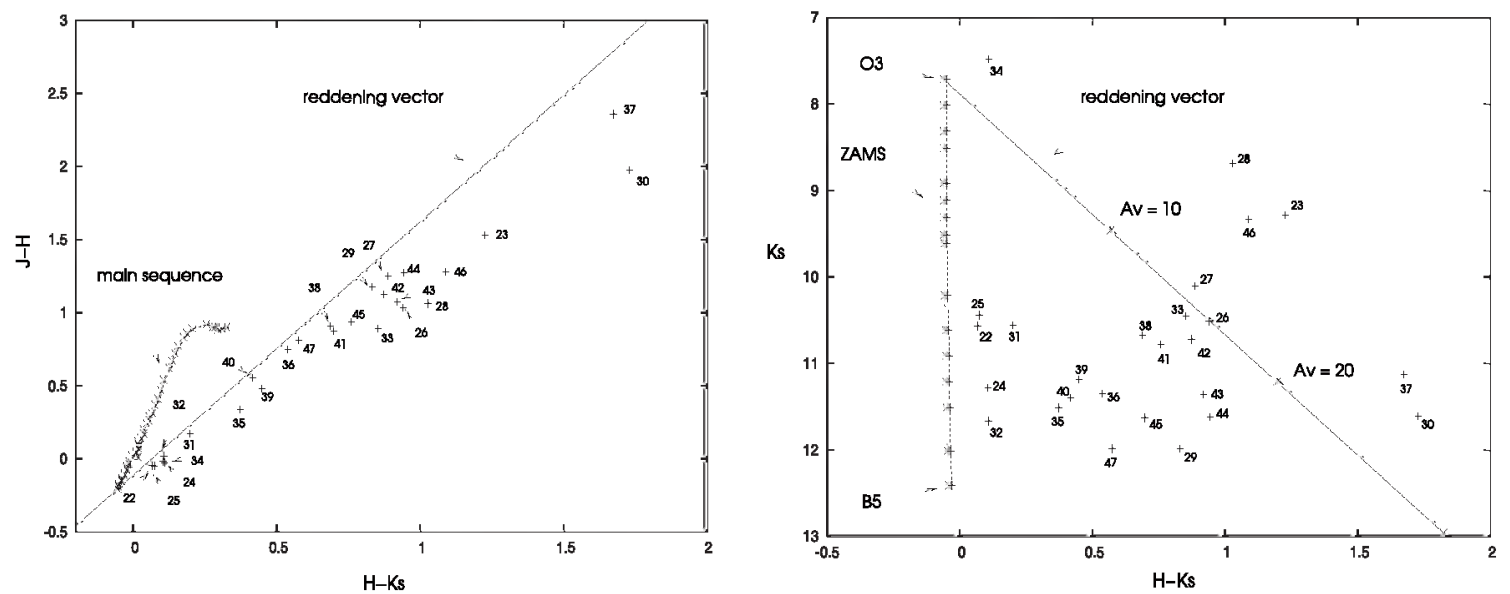

Fig. 10. Color-color and magnitude-color diagrams showing the 2MASS sources with infrared excess.

radio source at $843 \mathrm{MHz}$ (see Fig. 2) and with a bright source in the MSX band $\mathrm{E}$ (see Fig. 7), showing that a compact $\mathrm{H}$ II region may be present inside the molecular cloud. The MSX sources \#20 and \#21 coincide with a small source detected in both bands $A$ and $E$ (see Fig. 7) and with a point radio source detected at $843 \mathrm{MHz}$ (see Fig. 2). The detection of radio continuum emission is compatible with the classification of compact H II region of source \#20. The fact that IR emission was detected in both bands $A$ and $E$ suggests contributions of warm interstellar dust and/or ionized gas. The radio source coincident with source \#19 is about $48^{\prime \prime} \times 38^{\prime \prime}$ in size and its flux density is about $30 \mathrm{mJy}$. No electron density was estimated since, very probably, the compact $\mathrm{H}$ II region is optically thick at this low frequency. These results reinforce the idea that this is a very active star forming region.

Particularly interesting is the bright rim region at $(l, b) \simeq$ $\left(286^{\circ} 10^{\prime},-0^{\circ} 10^{\prime}\right)$. The 2 MASS sources \#27, \#28, and \#29, and the MSX source \#13 are located in this area, projected onto the molecular envelope. IRAS sources \#3 and \#5 are also placed close to the border of the HII region, coincident with molecular material. Source \#3 coincides with a region emitting in the MSX band A and with core 2 .

A bunch of protostellar objects is almost coincident in position with the open cluster NGC 3324: three MYSOs (MSX sources \#14,\#15, and \#17), and the 2MASS sources \#32, \#33, and \#34. The color-color diagram shows that two of these sources have relatively small IR excess. Some of these sources coincide with the loose IR cluster IC 2599 listed by Dutra et al. (2003). These facts suggest that stellar formation is ongoing in the region of NGC 3324, as previously found by Carraro et al. (2001). Also IRAS sources \#6 and \#10 are projected onto the dense cores 5 and 7 , respectively. The large luminosities $L_{\text {IRAS }}$ estimated for some of these sources suggest that they are protostellar candidates for massive stars or star clusters.

The sources IRAS 10355-5828 (286 $\left.16.92,-0^{\circ} 15.6\right)$ and MSX G286.2868-00.2604 (neither included in Table 3 nor shown in Fig. 9) coincide with HD 92207, a red supergiant whose membership to the open cluster is a matter of debate (Carraro et al. 2001). Its MSX fluxes correspond to an evolved object.

The detection of protostellar candidates in the IRAS, MSX, and 2MASS databases, strongly indicates that active stellar formation is currently ongoing in the molecular shell around Gum 31 .

To sum up, most of the protostellar candidates detected towards Gum 31 appear projected onto the shell detected in the
${ }^{12} \mathrm{CO}$ line, and coincide with the dense cores detected in $\mathrm{C}^{18} \mathrm{O}$ emission. Some are located close to the bright sharp borders of the H II region, and near the open cluster NGC 3324.

The presence of protostellar objects on the molecular envelope bordering the ionized region indicates that star formation has been triggered by the expansion of the H II region. The distribution of the molecular and $\mathrm{HI}$ gas around the ionized region suggests that star formation could be due to the collect and collapse process.

\section{Summary}

We have analyzed the interstellar medium in the environs of the $\mathrm{H}$ II region Gum 31 to investigate the action of the massive stars in the exciting open cluster NGC 3324 on the surrounding neutral material.

We based our study on H I $21-\mathrm{cm}$ line emission data belonging to the SGPS, radio continuum data at $0.843,2.4$ and $4.85 \mathrm{GHz}$ from the PMN Southern Radio Survey, ${ }^{12} \mathrm{CO}$ data from Yonekura et al. (2005), and IRAS (HIRES) and MSX data.

Adopting a distance of $3.0 \pm 0.5 \mathrm{kpc}$, we have derived an ionized mass of $3300 \pm 1100 M_{\odot}$ and an electron density of $33 \pm 3 \mathrm{~cm}^{-3}$. The four O-type stars in the HD 92206 multiple system can provide the necessary UV photon flux to maintain the $\mathrm{H}$ II region ionized.

The HI emission distribution in the environs of Gum 31 shows the presence of an $\mathrm{H}$ I shell approximately centered at the position of the multiple system HD 92206. The H I shell closely encircles the optical nebula. It is detected within the velocity range -32 to $-13 \mathrm{~km} \mathrm{~s}^{-1}$ and its systemic velocity of $-23 \mathrm{~km} \mathrm{~s}^{-1}$ is coincident, within errors, with the velocity of the ionized gas in the nebula $\left(-18 \mathrm{~km} \mathrm{~s}^{-1}\right)$. The H I structure is $10.0 \pm 1.7 \mathrm{pc}$ in radius and expands at about $11 \mathrm{~km} \mathrm{~s}^{-1}$. The associated atomic mass is $1500 \pm 500 M_{\odot}$.

Molecular gas with velocities in the range -27.2 and $-14.0 \mathrm{~km} \mathrm{~s}^{-1}$ surrounds the brightest parts of Gum 31. The sharp interface between the ionized and molecular material indicates that these gas components are interacting. We have estimated a molecular mass of $(1.1 \pm 0.5) \times 10^{5} M_{\odot}$. Two possible origins can be suggested for the molecular shell: 1) it could have originated in the collect and collapse process driven by the expansion of the H II region; or 2) it could represent the remains of the natal cloud where the open cluster NGC 3324 formed. In both cases, a large amount of the neutral hydrogen in the $\mathrm{H}$ I shell may have originated in the photodissociation of the molecular gas. 
The volume density of the molecular cloud, higher than the electron density $\left(33 \mathrm{~cm}^{-3}\right)$, implies that the $\mathrm{HII}$ region is expanding.

The emission in the far infrared correlates with the HII region and the $\mathrm{H}$ I envelope, indicating that the observed emission is probably related to the neutral and molecular envelopes.

The distribution of the emission in the MSX band A, which closely delineates the $\mathrm{HII}$ region and correlates with the molecular emission, suggests the presence of a PDR at the interface between the ionized and molecular gas.

A number of MSX, IRAS, and 2MASS point sources with IR spectra compatible with protostellar objects appear projected onto the molecular envelope, implying that stellar formation is active in the higher density cores of the molecular envelope around Gum 31, where massive stars or star clusters are probably being formed.

The optical image of the nebula does not show clear evidence of a central cavity, as expected in a stellar wind bubble, suggesting that the massive stars in the cluster have weak stellar winds or existed for a short period of time to develop an interstellar bubble in a high density interstellar medium.

Acknowledgements. We dedicate this paper to our dear friend and collaborator Prof. Virpi Niemela, who passed away on December 18th, 2006. C.E.C. is extremely grateful to her for her teaching and encouragement, and mainly for years of friendship. We thank the referee for many important comments and suggestions which largely improved this presentation. We also thank Dr. Y. Yonekura for making his $\mathrm{CO}$ data available to us. This project was partially financed by the Consejo Nacional de Investigaciones Científicas y Técnicas (CONICET) of Argentina under project PIP 5886/05 and PIP 5697/05, Agencia PICT 14018, and UNLP under projects 11/G072 and 11/G087. The Digitized Sky Survey (DSS) was produced at the Space Telescope Science Institute under US Government grant NAGW-2166.

\section{References}

Brand, J., \& Blitz, L. 1993, A\& A, 275, 67

Cappa, C. E., Niemela, V. S., Martín, M. C., \& McClure-Griffiths, N. M. 2005. A\&A, 436, 155

Carraro, G., Patat, F.. \& Baumgardt. H. 2001. A\&A. 371. 107

Caswell, J. L., \& Haynes, R. F. 1987, A\&A, 171, 261

Clariá, J. J. 1977, A\&A, 27, 145

Condon. J. J., Griffith, M. R., \& Wright, A. E. 1993, AJ. 106, 1095

Cesarsky, D., Lequeux, J., Abergel, A., et al. 1996, A\&A, 315, 305

Chlebowski T., \& Garmany C. D. 1991. ApJ, 368, 241
Cichowolski. S., Pineault. S., Arnal. E. M., et al. 2001. AJ. 122, 1938 Comerón. F., Schneider, N., \& Russeil, D. 2005, A\&A. 433, 955

Cutri, R. M., Skrutskie. M. F., van Dyk. S.. et al. 2003. The IRSA 2MASS AllSky Point Source Catalog. NASA/IPAC Infrared Science Archive

Dale, J. E., Bonnell, I. A., \& Whitworth, A. P. 2007, MNRAS, 375, 1291

Deharveng. L.., Zavagno, A.., Salas, L.., et al. 2003. A\&A. 399, 1135

Deharveng. L.. Zavagno, A., \& Caplan., J. 2005. A\&A. 433, 565

Digel S. W.. Hunter S. D.. \& Mukherjee R. 1995. ApJ. 441. 270

Duncan, A. R., Stewart, R. T., Haynes, R. F., \& Jones, K. L. 1995, MNRAS, 277. 36

Dutra, C. M., Bica, E., Soares, J., \& Barbuy, B. 2003, A\&A., 400, 533

Egan. M. P., Price, S. D., Moshir, M. M., et al. 1999. The Midcourse Space Experiment Point Source Catalog Version 1:2 Explanatory Guide. AFRLVS-TR-1999-1522. Air Force Research Laborartory

Elmegreen. B. G.. \& Lada, C. J. 1977. ApJ, 214. 725

Georgelin. Y. M., Lortet, M. C., \& Testor, G. 1986, A\&A, 162, 265

Gum. C. S. 1955, MmRAS, 67, 155

Hanson. M. M.. Howarth. I. D.. \& Conti. P. S. 1997. ApJ. 489. 698

Hildebrand. R. H. 1983, Q. J. RAS, 24, 267

Hosokawa, T., \& Inutsuka, S. 2005, ApJ, 623,917

Hosokawa, T., \& Inutsuka, S. 2006, ApJ, 646, 240

Jeffers. H. M., van den Bos. W. H., \& Greeby, F. M. 1963, Publ. Lick Obs., 21

Junkes. N., Fürst. E.. \& Reich. W. 1992. A\&A. 261. 289

Koornneef, J. 1983, A\&A. 128, 84

Lozinskaya, T. A. 1982, Astrophys. Space Sci., 87, 313

Lumsden. S. L.., Hoare, M. G., Oudnaidjer, R. D., \& Richards, D. 2002. MNRAS, 336, 621

Martins. F.. Schaerer. D.. \& Hillier. D. J. 2005. A\&A. 436. 1049

Mathys 1988, A\&AS, 76, 427

McClure-Griffiths. N. M., Dickey, J. M., Gaensler, B. M., et al. 2005, ApJS, 158. 178

Mezger, P. D., \& Henderson. A. P. 1967, ApJ, 147, 471

Moffat, A. F. J., \& Vogt. N. 1975, A\&S, 20, 125

Parker. Q. A.. Phillipps, S.. Pierce, M. J.. et al. 2005, MNRAS. 362. 689

Prinja, R. K., Barlow, M. J., \& Howarth, I. D. 1990, ApJ, 361, 607

Rohlfs, K. 1986. Tools of Radioastronomy (Berlin. Heidelberg: SpringerVerlag). 159

Romero. G. A. 2006. Ph.D. Thesis

Sadler, E. M.. \& Hunstead. R. W. 2001. ASP Conf. Ser.. 232. 53

Spitzer, L. 1978, Physical Processes in the Interstellar Medium (New York: Wiley-Interscience publication)

Vazquez. R. A., \& Feinstein. A. 1990. Rev. Mex. Astron. Astrofis., 21. 346

Walborn. N. 1982. AJ. 87. 1300

Walborn. N. 1995, in The $\eta$ Car region: a laboratory of Stellar Evolution, ed. Niemela, V., Morrell. N., \& Feinstein. A. 1995, Rev. Mex., Conf. Ser., 2, 51 Weaver, R., McCray, R., Castor, J., Shapiro, P., \& Moore, R. 1977, ApJ, 218, 377 Wood. D. O. S.. \& Churchwell, E. 1989. ApJS. 69. 831

Yamaguchi. R.. Saito. H.. \& Mizuno. N. 1999. PASJ. 51.791

Whitworth. A. P.. Battal, A. S.. Chapman. S. J.. Disney. M. J., \& Turner. J. A. 1994, A\&A, 290, 421

Yonekura, Y.. Asayama. S., \& Kimura, K. 2005, ApJ. 634. 476 